\title{
Gain dependence of measured spectra in coherent Brillouin optical time-domain analysis sensors
}

\author{
Jon Mariñelarena, Javier Urricelqui, Alayn Loayssa* \\ Universidad Pública de Navarra, Campus Arrosadía s/n, 31006, Pamplona, Spain
}

\begin{abstract}
We report on the effects of large pump pulse powers on Brillouin optical time-domain analysis (BOTDA) sensors based on phase-modulated probe wave and coherent detection. It is found that the large Brillouin gain that comes from the use of high power pulses induces a narrowing of the RF phase-shift spectrum that is measured in these sensors. This narrowing leads to a Brillouin frequency shift measurement error when the sensor is configured for dynamic measurements. However, the effect has been found to be less significant than that observed in dynamic slope-assisted BOTDA sensors based on amplitude.
\end{abstract}

Keywords: Brillouin scattering, Brillouin optical time-domain analysis, coherent sensors, dynamic sensing

\section{INTRODUCTION}

New techniques have been proposed in the last years to extend the Brillouin optical time-domain analysis (BOTDA) sensors to dynamic measurements. ${ }^{1-3}$ The objective is to be able to measure fast strain and temperature variations with high precision and resolution satisfying the increasing demand for distributed fiber sensors applied to structural health monitoring applications.

The first dynamic measurement techniques were based on the frequency discriminator principle, the so-called slope-assisted BOTDA, where changes of the Brillouin frequency shift (BFS) were translated to changes of the detected probe power by the slope of the Brillouin gain spectrum (BGS). ${ }^{1,2}$ However, recently, a previously unknown effect in stimulated Brillouin scattering (SBS) interaction has been discovered, by which the linewidth of the BGS has been found to depend on the pump pulse power. ${ }^{4}$ This unexpected finding means that BFS measurement error can appear in techniques such as the slope-assisted BOTDA that rely on the shape of the BGS. ${ }^{5}$

We have recently proposed a dynamic BOTDA sensor tolerant to Brillouin gain changes in the sensing fiber. ${ }^{3}$ This technique is based on the measurement of the phase-shift spectra of the RF signal that results from the coherent detection of a phase-modulated probe wave. The RF phase-shift is found to be dependent just on the BFS experienced by the probe wave, but not on the pump pulse power for the range of Brillouin gains normally used in BOTDA sensors. In this work, we study the Brillouin gain dependence of the RF phase-shift spectrum that is measured in these coherent BOTDA systems when high pump pulse powers are deployed. Moreover, we experimentally confirm the existence of the Brillouin linewidth broadening for large pump pulses in conventional BOTDA systems. ${ }^{4}$ Furthermore, we compare both methods, conventional BOTDA and coherent BOTDA, in terms of the amount of spectrum shape change with increasing Brillouin gain.

\section{BRILLOUIN LINEWIDTH DEPENDENCE IN CONVENTIONAL AND COHERENT BOTDA SYSTEMS}

The fundamentals of dynamic sensors using coherent BOTDA are shown in Fig. 1(a). ${ }^{3}$ A phase-modulated probe wave is injected in one end of an optical fiber while a pump pulse is introduced at the other end. This probe wave interacts with the pump pulse via stimulated Brillouin scattering. If SBS interaction only affects the upper sideband of the phase-modulated wave, the probe wave power detected in the photodetector at the modulation frequency, $f_{R F}$, as a result of the interaction with the pulse at a particular location, $\mathrm{z}$, can be expressed as: ${ }^{3}$

$$
\begin{aligned}
\left.P(t)\right|_{f_{R F}} & =2 E_{0} E_{S B}\left[G_{S B S} \cos \left(2 \pi f_{R F} t+\varphi_{S B S}\right)-\cos \left(2 \pi f_{R F} t\right)\right] \\
& \approx \frac{2 E_{0} E_{S B} g_{0} \Delta \nu_{B}}{\sqrt{\Delta \nu_{B}^{2}+(2 \Delta \nu)^{2}}} \cos \left(2 \pi f_{R F} t-\arctan \left(2 \frac{\Delta \nu}{\Delta \nu_{B}}\right)\right)
\end{aligned}
$$


where $G_{S B S}$ and $\varphi_{S B S}$ are the magnitude and phase-shift of the Brillouin gain spectrum, $E_{S B}$ and $E_{0}$ are the complex amplitude of the carrier and sidebands of the phase-modulated probe wave and $g_{0}$ is the peak of the Brillouin gain spectrum. $\Delta \nu_{B}$ is the Brillouin linewidth and $\Delta \nu$ is the detuning of the probe wave frequency from the maximum of the Brillouin interaction at the BFS. Small gain $g_{0}$ and Lorentzian spectral shape are assumed to obtain the last approximation term in Eq. 1. Note that, under this approximation, the RF phase-shift of the detected signal is independent of the peak Brillouin gain. However, it is necessary to further investigate if the $\mathrm{RF}$ phase-shift measured in coherent BOTDA system is also gain dependent for large gain.

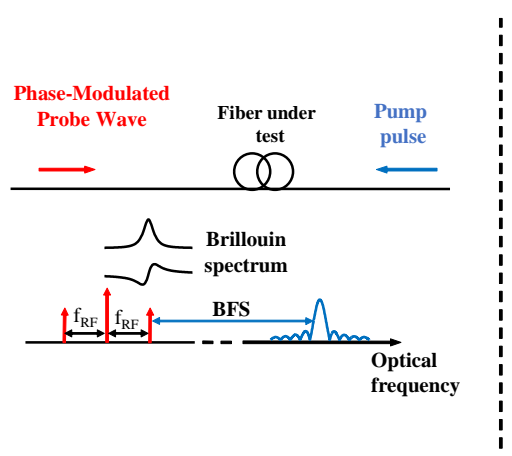

(a)

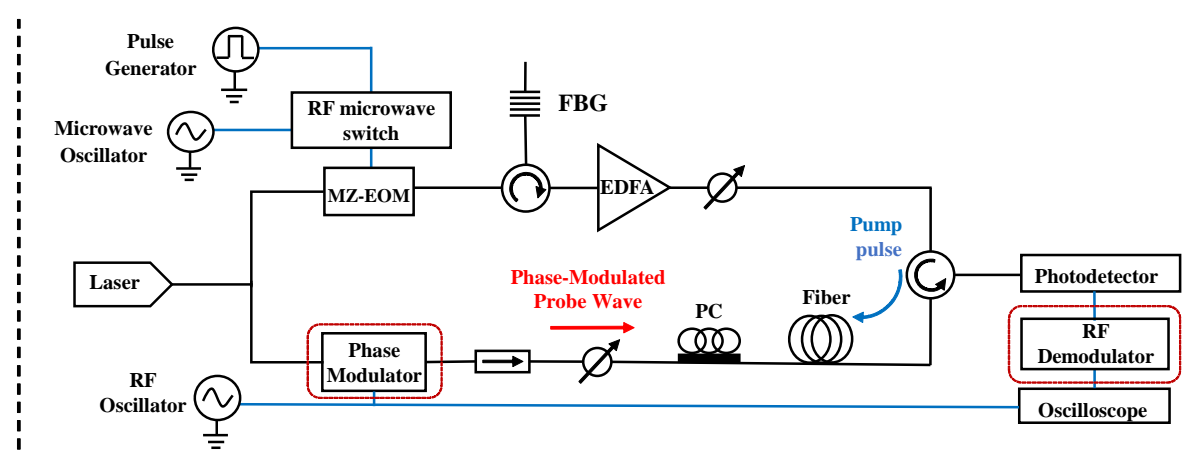

(b)

Figure 1. (a) Schematic representation of SBS interation. (b) Experimental setup for the coherent BOTDA dynamic system. The components that are removed for conventional BOTDA measurements are highlighted in red boxes.

Fig. 1(b) depicts the experimental setup deployed in this investigation. ${ }^{3}$ In the upper branch, optical pump pulses are formed with a Mach-Zehnder electro-optic modulator (MZ-EOM) biased at minimum and driven by microwave pulses. One of the two pump sidebands generated is filtered with a fiber Bragg gratting (FBG) and amplified by a high-power erbium doped fiber amplifier (EDFA). Then, an optical attenuator controls the pump peak power injected to the fiber, which can reach a peak value of $4 \mathrm{~W}$. On the lower branch, the probe wave is generated with a phase modulator driven by a $1.3-\mathrm{GHz} \mathrm{RF}$ signal. This modulated probe wave is injected into the fiber whose polarization state is adjusted to optimize the Brillouin interaction along $30-\mathrm{m}$ of single-mode fiber (SMF-28). The resultant probe wave is detected in a receiver, and, finally, the RF signal is demodulated and captured in a digital oscilloscope.

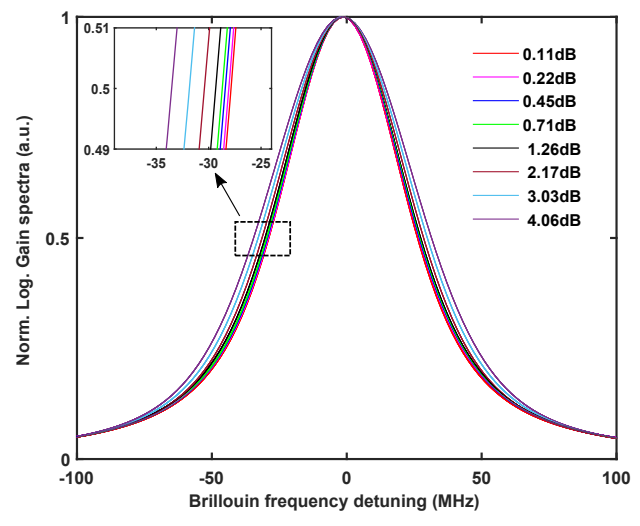

Figure 2. Experimental Brillouin gain spectra for 20-ns pulse duration when gain increases from 0.1 to $4 \mathrm{~dB}$.

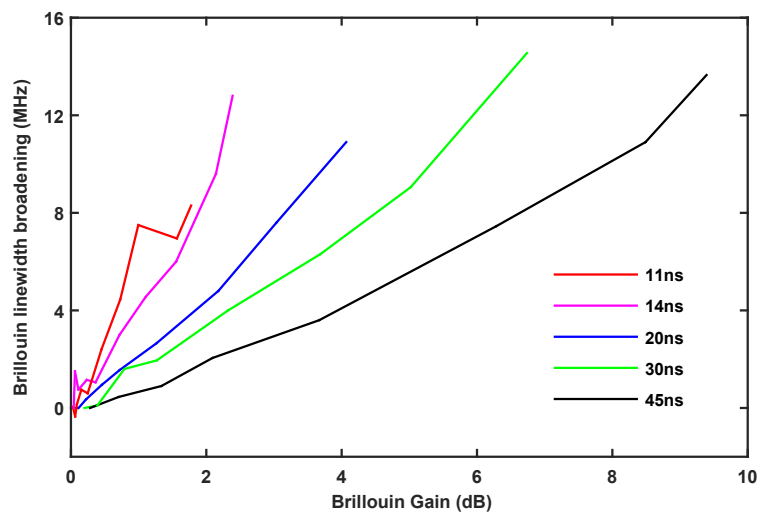

Figure 3. Brillouin linewidth broadening for different pulse lengths when gain is risen.

We started by confirming experimentally the existence of the gain dependence of the Brillouin linewidth in conventional BOTDA systems. ${ }^{4}$ Notice that the setup in Fig. 1(b) can be used for conventional BOTDA measurements by simply removing the phase modulator and RF demodulation module. Brillouin linewidth broadening for 20-ns pump pulses when gain is risen up to $4.06 \mathrm{~dB}$ is shown in Fig. 2. These gain values are 
achieved injecting peak pulse powers from $20 \mathrm{dBm}$ to $36 \mathrm{dBm}$, respectively. The same broadening effect has been observed for different pulse durations, as it is summarized in Fig. 3. The general trends and linewidth broadening agree well with the results presented, ${ }^{4}$ and hence independently confirming the existence of this effect.

Then, we focused our research on studying the effect that Brillouin gain may cause on the RF phase-shift spectrum detected using the coherent BOTDA system. Experimental RF phase-shift spectra of 20-ns pulses are shown in Fig. 4 for increasing peak Brillouin gain. In this case, a gain dependence of the RF phase-shift spectrum shape is also observed. However, the resultant measured spectra get narrower when gain is increased, contrary to conventional BOTDA systems response. For instance, the phase-shift spectrum for 20-ns pulse narrows $0.7 \mathrm{MHz}$ when gain is increased to $1 \mathrm{~dB}$. Narrowing has been also measured at the frequencies corresponding to the the full wave half maximum (FWHM) of the Brillouin gain for different pulse durations (11 ns, $14 \mathrm{~ns}, 20 \mathrm{~ns}, 30 \mathrm{~ns}$ and $45 \mathrm{~ns}$ ) as depicted in Fig. 5. Furthermore, the comparison of Fig. 3 and Fig. 5 demonstrate that the gain influence on RF phase-shift spectrum measured in coherent BOTDA systems is smaller than on the Brillouin gain spectrum measured in conventional systems.

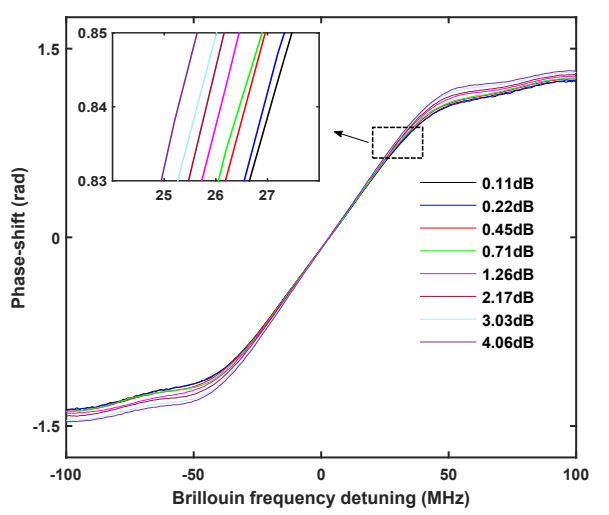

Figure 4. Experimental RF phase-shift spectra for 20-ns pulse when gain increases from 0.1 to $4 \mathrm{~dB}$.

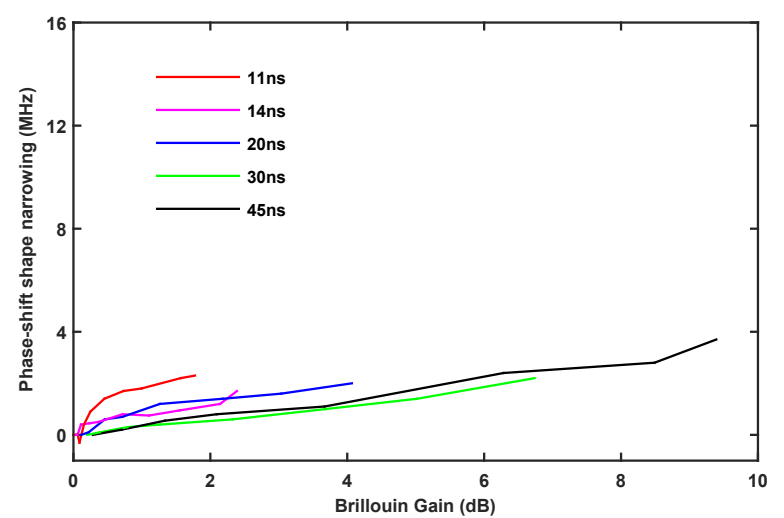

Figure 5. RF phase-shift shape narrowing for different pulse lenghts when gain is risen.

The unexpected RF phase-shift spectrum narrowing (instead of broadening) on coherent BOTDA systems was also analyzed with the model presented in Eq. 1. We used the first term of Eq. 1 (without small-gain approximation) and modeled the Brillouin gain spectrum as the convolution of a Lorentzian and Gaussian profiles to take into account the broadening when using short pulses. Fig. 6 shows the calculated RF phase-shift spectrum as a function of gain for 20-ns pulses. These calculations assume that there is no gain dependence of the Brillouin broadening; hence, the observed change in the spectrum shape (narrowing) is just due to the nature of the interference between detected waves that is conveyed by Eq. 1. However, the observed narrowing of the spectra in Fig. 6 is larger than that observed in the experimental results in Fig. 4.

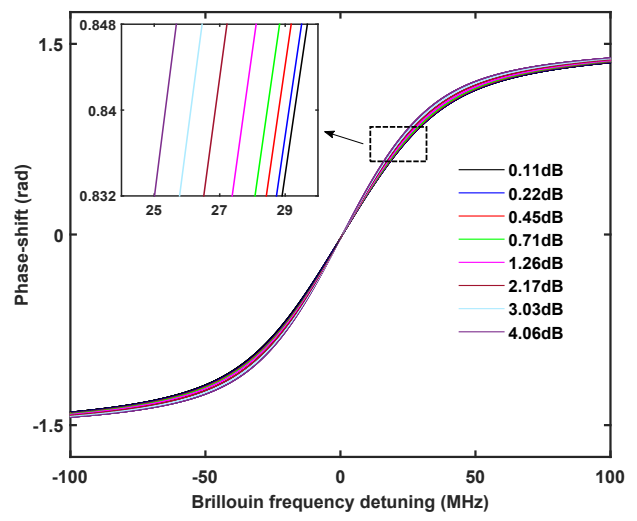

Figure 6. Simulated phase-shift narrowing for 20-ns pulse considering only gain increment.

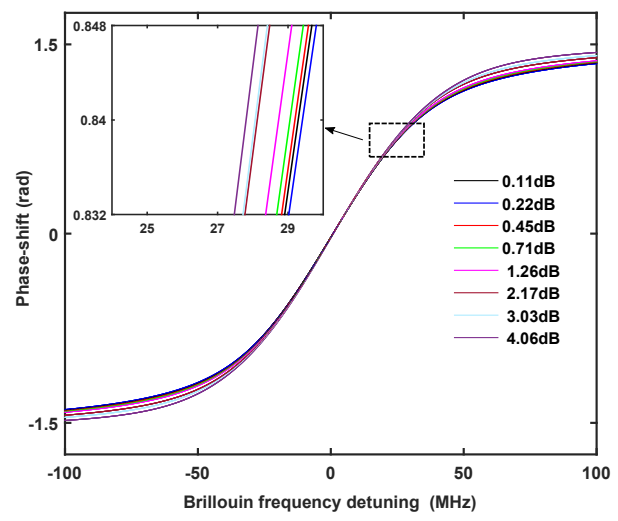

Figure 7. Simulated phase-shift narrowing for 20-ns pulse considering gain increment and linewidth broadening. 
Therefore, we repeated the calculations, but this time incorporating the Brillouin linewidth broadening with increasing gain. These new results, which are shown in Fig. 7, highlight a reduced narrowing of the spectra, which agrees with the trends in the experiments. Therefore, we can conclude than in coherent BOTDA the natural trend to narrowing of the RF phase-shift spectra for increasing gain is compensated to some extend by the linewidth broadening effect. This is the reason that the gain dependence of shape of the spectra is less important in these systems that in conventional BOTDA systems.

Finally, we analyzed the measurement error brought by the observed RF phase-shift narrowing in coherent BOTDA for dynamic sensing. Fig. 8 depicts the error, calculated from the experimental measurement of RF phase-shift spectrum narrowing, for 20-ns pulses when Brillouin gain is modified. For instance, if a maximum error of $1 \mathrm{MHz}$ is desired in the measurements, we could tolerate a $1.1 \mathrm{~dB}$ Brillouin gain on the probe wave, which means that up to $30.5 \mathrm{dBm}$ pump peak pulse power may be injected to the fiber without exceeding this threshold.

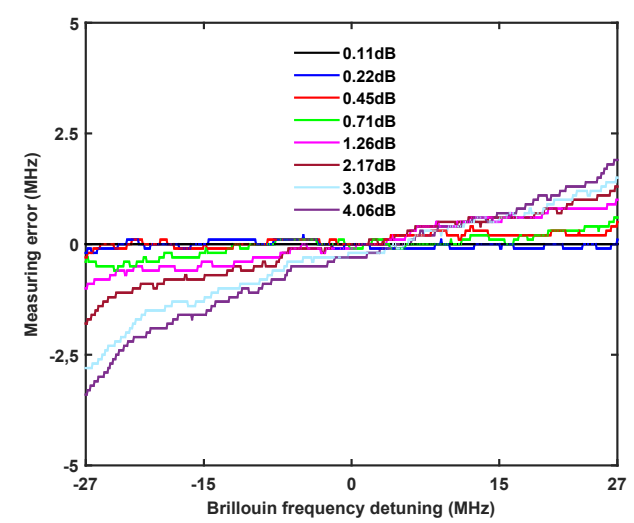

Figure 8. Measuring error in coherent BOTDA system for 20-ns pulses when Brillouin gain is modified.

\section{CONCLUSIONS}

In summary, this work demonstrates that the RF phase-shift spectrum measured on coherent BOTDA sensors based on a phase-modulated probe wave is gain dependent, but, in any case, this dependence is less important than in conventional BOTDA sensors. Furthermore, this effect has significance only in very short fibers, because for longer lengths, nonlinear effects such as modulation instability, self-phase modulation or Raman scattering impose a more stringent limitation to the pump power that can be deployed.

\section{ACKNOWLEDGMENTS}

The authors wish to acknowledge the financial support from the Spanish Ministerio de Economía y Competitividad through the project TEC2013-47264-C2-2-R, FEDER funds and Universidad Pública de Navarra.

\section{REFERENCES}

1. R. Bernini, A. Minardo, and L. Zeni, "Dynamic strain measurement in optical fibers by stimulated Brillouin scattering," Opt. Letters 34(17), pp. 2613-2615, (2009).

2. Y. Peled, A. Motil, L. Yaron, and M. Tur, "Slope-assisted fast distributed sensing in optical fibers with arbitrary Brillouin profile," Opt. Express 19(21), pp. 19845-19854, (2011).

3. J. Urricelqui, A. Zornoza, M. Sagues, and A. Loayssa, "Dynamic BOTDA measurements based on Brillouin phase-shift and RF demodulation.," Opt. Express 20(24), pp. 26942-9, (2012).

4. A. Motil, R. Hadar, I. Sovran, and M. Tur, "Gain dependence of the linewidth of Brillouin amplification in optical fibers," Opt. Express 22(22), pp. 27535-27541, (2014).

5. A. Motil, R. Hadar, I. Sovran, and M. Tur, "The effect of the gain dependency of the line width of Brillouin amplification on double slope-assisted dynamic sensing techniques," Proc. SPIE 9634, (2015). 
Copyright 2016 Society of Photo Optical Instrumentation Engineers. One print or electronic copy may be made for personal use only. Systematic electronic or print reproduction and distribution, duplication of any material in this paper for a fee or for commercial purposes, or modification of the content of the paper are prohibited. 